\title{
On the Factorization of Differential Modules
}

\author{
Min $\mathrm{Wu}$
}

\begin{abstract}
Differential modules are modules over rings of linear (partial) differential operators which are finite-dimensional vector spaces. We present a generalization of the Beke-Schlesinger algorithm that factors differential modules. The method requires solving only one set of associated equations for each degree $d$ of a potential factor.
\end{abstract}

Mathematics Subject Classification (2000). 13N05; 13N10; 13N15; 16S32; 16S36; 32C38; 33F10; 68W30.

Keywords. Differential (sub)modules, rings of linear differential operators, integrable systems, hyperexponential solutions.

\section{Introduction}

The problem of factoring linear ordinary differential equations or operators has been studied for a long time since Beke [1] and Schlesinger [9] introduced the idea of associated equations and developed a factorization algorithm for linear ordinary differential equations (operators). This idea together with some improvements $[2,10]$ on the Beke-Schlesinger algorithm inspired the study of reducing the factorization problem to that of finding hyperexponential solutions of associated systems. Making use of the main algorithm in [5] for computing hyperexponential solutions of systems of linear partial differential equations (PDE's), Li et al $[6,7]$ generalize the Beke-Schlesinger factorization algorithm to systems of linear PDE's (in one or several unknowns) with finite-dimensional solution spaces. However, in their algorithm, there is a combinatorial explosion caused by trying out all the possible sets of leading derivatives for a potential factor. To avoid this combinatorial explosion, we formulate the factorization problem in terms of differential modules and then reduce the factorization problem to that of finding one-dimensional differential submodules, and further to that of computing hyperexponential solutions of associated systems.

Throughout this paper, fields are always assumed to be commutative, modules and vector spaces are left modules and left vector spaces. The notation $(\cdot)^{\tau}$ 
denotes the transpose of vectors or matrices. We write $\mathrm{M}_{n}(k)$ (resp. $\mathrm{GL}_{n}(k)$ ) for the set of all $n \times n$ (resp. invertible) matrices with entries in a field $k$.

The paper is organized as follows. Section 2 introduces the notion of differential modules and gives some straightforward generalizations of results in Chapter 1 and 2 of [8]. Section 3 outlines the idea of the generalized Beke-Schlesinger factorization method, by reducing the problem of finding $d$-dimensional submodules of a differential module to that of finding 1-dimensional submodules of its exterior power and by linking 1-dimensional submodules and hyperexponential solutions of the associated integrable systems. Section 4 describes a factorization algorithm for differential modules.

\section{Preliminaries}

\subsection{Differential Modules}

Let $k$ be a field (of characteristic zero).

Definition 2.1. A derivation on the field $k$ is a map $\delta: k \mapsto k$ satisfying

$$
\delta(a+b)=\delta(a)+\delta(b), \quad \delta(a b)=\delta(a) b+a \delta(b),
$$

for all $a, b \in k$.

The field $k$ equipped with a set of commuting derivations $\delta_{1}, \ldots, \delta_{m}$ is called a (partial) differential field. An element $c$ of $k$ is called a constant w.r.t. $\delta_{i}$ if $\delta_{i}(c)=0$. An element $c$ of $k$ is called a constant if $c$ is a constant w.r.t. all $\delta_{i}$ 's. All constants of $k$ form a subfield of $k$, which we denote by $C$.

Let $k$ be a differential field equipped with the derivations $\delta_{1}, \ldots, \delta_{m}$. The ring of linear differential operators over $k$ is the ring $k\left[\partial_{1}, \ldots, \partial_{m}\right]$ of noncommutative polynomials in $\partial_{1}, \ldots, \partial_{m}$ with the following multiplicative rules:

$$
\partial_{i} \partial_{j}=\partial_{j} \partial_{i} \quad \text { and } \quad \partial_{i} a=a \partial_{i}+\delta_{i}(a)
$$

for all $a \in k$ and any $i, j \in\{1, \ldots, m\}$. Denote $\mathbb{D}=k\left[\partial_{1}, \ldots, \partial_{m}\right]$. Definition D.3 in [8] states that $M$ is a differential module if $M$ is a $\mathbb{D}$-module that is also a finite-dimensional vector space over $k$. Hence, the dimension of the differential module $M$ is understood as the dimension of $M$ as a vector space over $k$.

\subsection{Constructions on Differential Modules}

We observe that the notion of ordinary differential modules discussed in Chapter 1 and 2 of [8] is a special case of differential modules where $m=1$. As a straightforward generalization, the constructions on differential modules can be carried on as follows.

A (differential) submodule $N$ of $M$ is a $k$-vector subspace $N \subseteq M$ such that $\partial_{i}(N) \subseteq N$ for $i=1, \ldots, m$.

Let $N$ be a submodule of $M$. Then, $M / N$ endowed with the map $\partial_{i}$ given by

$$
\partial_{i}(w+N)=\partial_{i}(w)+N,
$$


for $w \in M$ and $i=1, \ldots, m$, is the quotient differential module.

The direct sum of two differential modules $M_{1}$ and $M_{2}$ is $M_{1} \oplus M_{2}$ endowed with the map $\partial_{i}$ given by

$$
\partial_{i}\left(w_{1}+w_{2}\right)=\partial_{i}\left(w_{1}\right)+\partial_{i}\left(w_{2}\right)
$$

for $w_{1} \in M_{1}, w_{2} \in M_{2}$ and each $i$.

The tensor product $M_{1} \otimes M_{2}$ of two differential modules is $M_{1} \otimes_{k} M_{2}$ endowed with the map $\partial_{i}$ given by

$$
\partial_{i}\left(w_{1} \otimes w_{2}\right)=\partial_{i}\left(w_{1}\right) \otimes w_{2}+w_{1} \otimes \partial_{i}\left(w_{2}\right),
$$

for $w_{1} \in M_{1}, w_{2} \in M_{2}$ and each $i$.

The $d$-th exterior power $\wedge^{d} M$ of a differential module $M$ is the $k$-vector space $\wedge_{k}^{d} M$ endowed with the map $\partial_{i}$ given by

$$
\partial_{i}\left(w_{1} \wedge \cdots \wedge w_{d}\right)=\sum_{j=1}^{d} w_{1} \wedge \cdots \wedge \partial_{i}\left(w_{j}\right) \wedge \cdots \wedge w_{d}
$$

for $w_{1}, \ldots, w_{d} \in M$ and each $i$.

A morphism $\phi: M_{1} \rightarrow M_{2}$ is a $k$-linear map $\phi$ such that $\phi \circ \partial_{i}=\partial_{i} \circ \phi$ for $i=1, \ldots, m$, that is, $\phi$ is a $\mathbb{D}$-linear map.

Two differential modules are said to be isomorphic if there exists a bijective morphism between them.

The internal Hom, $\operatorname{Hom}\left(M_{1}, M_{2}\right)$ of two differential modules is the $k$-vector space $\operatorname{Hom}_{k}\left(M_{1}, M_{2}\right)$ of all $k$-linear maps from $M_{1}$ to $M_{2}$ endowed with the map $\partial_{i}$ on $\operatorname{Hom}_{k}\left(M_{1}, M_{2}\right)$ given by

$$
\partial_{i}(\ell)\left(w_{1}\right)=-\ell\left(\partial_{i}\left(w_{1}\right)\right)+\partial_{i}\left(\ell\left(w_{1}\right)\right),
$$

for all $\ell \in \operatorname{Hom}_{k}\left(M_{1}, M_{2}\right)$ and $w_{1} \in M_{1}$. A special case of the internal Hom is the dual module of a differential module $M$ defined to be $M^{*}=\operatorname{Hom}_{k}\left(M, \mathbf{1}_{\mathbf{k}}\right)$ where $\mathbf{1}_{\mathbf{k}}$ denotes the differential module $k w$ with $\partial_{i}(w)=0$ for $i=1, \ldots, m$.

\section{Reduction of the Factorization Problem}

By factoring a differential module, we mean finding its proper submodules. In the sequel, we will outline the idea of the generalized Beke-Schlesinger algorithm for factoring differential modules.

\subsection{From $M$ to $\wedge^{d} M$}

We have the following generalization of Lemma 10 in [3] or the corresponding statements in Section 4.2.1 of [8]:

Proposition 3.1. Let $M$ be a differential module. Then, $M$ has a d-dimensional submodule if and only if $\wedge^{d} M$ has a 1-dimensional submodule generated by a decomposable element $u$, i.e., $u=w_{1} \wedge \cdots \wedge w_{d}$ with $w_{i} \in M$. 
Proof. Let $N$ be a $d$-dimensional submodule of $M$ with a basis $w_{1}, \ldots, w_{d}$ over $k$. Suppose that

$$
\partial_{i}\left(w_{1}, \ldots, w_{d}\right)^{\tau}=A_{i}\left(w_{1}, \ldots, w_{d}\right)^{\tau} \text { for } i=1, \ldots, m
$$

where $A_{i}=\left(a_{i s t}\right)_{1 \leq s, t<d} \in \mathrm{M}_{d}(k)$. Then, $\wedge_{k}^{d} N$ is a $k$-vector subspace of $\wedge_{k}^{d} M$ generated by $w_{1} \wedge \cdots \wedge w_{d}$. Moreover,

$$
\begin{aligned}
& \partial_{i}\left(w_{1} \wedge \cdots \wedge w_{d}\right)=\sum_{s=1}^{d} w_{1} \wedge \cdots \wedge \partial_{i}\left(w_{s}\right) \wedge \cdots \wedge w_{d} \\
= & \sum_{s=1}^{d} w_{1} \wedge \cdots \wedge\left(\sum_{t=1}^{d} a_{i s t} w_{t}\right) \wedge \cdots \wedge w_{d}=\operatorname{tr}\left(A_{i}\right)\left(w_{1} \wedge \cdots \wedge w_{d}\right) \in \wedge^{d} N,
\end{aligned}
$$

where $\operatorname{tr}\left(A_{i}\right)$ denotes the trace of the matrix $A_{i}$, for $i=1, \ldots, m$. So, $\wedge^{d} N$ is a 1-dimensional submodule of $\wedge^{d} M$.

Conversely, let $u \in \wedge^{d} M$ be a decomposable element which generates a 1dimensional submodule of $\wedge^{d} M$. Suppose that $u=w_{1} \wedge \cdots \wedge w_{d}$ with $w_{i} \in M$. Since $u \neq 0, w_{1}, \ldots, w_{d}$ are linearly independent over $k$ and there exists a basis $B$ of $M$ containing $w_{1}, \ldots, w_{d}$. Pick arbitrarily a finite number of distinct $b_{1}, \ldots, b_{s}$ in $B \backslash\left\{w_{1}, \ldots, w_{d}\right\}$. Since $w_{1}, \ldots, w_{d}, b_{1}, \ldots, b_{s}$ are linearly independent over $k$, so are $b_{1} \wedge u, \ldots, b_{s} \wedge u$. In particular, $b \wedge u \neq 0$ for any $b \in B \backslash\left\{w_{1}, \ldots, w_{d}\right\}$.

Consider a map $\phi_{u}: M \rightarrow \wedge^{d+1} M$ defined by $v \mapsto v \wedge u$. One can verify that $\operatorname{ker}\left(\phi_{u}\right)$ is a $k$-vector space. Let $v \in \operatorname{ker}\left(\phi_{u}\right)$. Then, $v \wedge u=0$ and

$$
0=\partial_{i}(v \wedge u)=\partial_{i}(v) \wedge u+v \wedge \partial_{i}(u)=\partial_{i}(v) \wedge u+v \wedge(a u) \quad \text { for some } a \in k,
$$

which implies that $\partial_{i}(v) \wedge u=0$ and $\partial_{i}(v) \in \operatorname{ker}\left(\phi_{u}\right)$ for each $i$. So, $\operatorname{ker}\left(\phi_{u}\right)$ is a $\mathbb{D}$-module.

Clearly, $\oplus_{i=1}^{d} k w_{i} \subseteq \operatorname{ker}\left(\phi_{u}\right)$. Suppose that $w \in \operatorname{ker}\left(\phi_{u}\right) \subset M$. Then, there exist $b_{1}, \ldots, b_{s} \in B \backslash\left\{w_{1}, \ldots, w_{d}\right\}$ such that

$$
w=\sum_{i=1}^{d} \lambda_{i} w_{i}+\sum_{j=1}^{s} \xi_{j} b_{j} \quad \text { with } \lambda_{i}, \xi_{j} \in k .
$$

Then, $0=w \wedge u=\sum_{j=1}^{s} \xi_{j}\left(b_{j} \wedge u\right)$. The linear independence of $b_{1} \wedge u, \ldots, b_{s} \wedge u$ therefore implies that $\xi_{j}=0$ for $j=1, \ldots, s$. So, $\operatorname{ker}\left(\phi_{u}\right)=\oplus_{i=1}^{d} k w_{i}$ is a $d$ dimensional submodule of $M$.

Proposition 3.1 converts the problem of finding $d$-dimensional submodules of a differential module $M$ into that of finding all those 1-dimensional submodules of $\wedge^{d} M$ whose generator is decomposable, and then reduces the factorization problem to its "subproblem" of finding 1-dimensional submodules.

Remark 3.2. Proposition 3.1 and the proof remain valid if $M$ is infinite-dimensional over $k$. 


\subsection{One-Dimensional Submodules of Differential Modules}

In this section, we will study how to find 1-dimensional submodules of differential modules.

First, we recall the definition of integrable systems given in Appendix D.1 of [8]: let $A_{1}, \ldots, A_{m}$ be $n \times n$ matrices with entries in a differential field $k$, the system

$$
\left\{\delta_{1}(Z)=A_{1} Z, \ldots, \delta_{m}(Z)=A_{m} Z\right\}
$$

is called an integrable system over $k$ of dimension $n$ if $A_{1}, \ldots, A_{m}$ satisfy the integrability conditions:

$$
\left[A_{i}, A_{j}\right]=\delta_{i}\left(A_{j}\right)-\delta_{j}\left(A_{i}\right), \quad \text { for any } i, j,
$$

where $\left[A_{i}, A_{j}\right]:=A_{i} A_{j}-A_{j} A_{i}$ is the commutator of the matrices $A_{i}$ and $A_{j}$.

Let $M$ be a differential module of dimension $n$. A choice of a basis $e_{1}, \ldots, e_{n}$ of $M$ satisfying

$$
\partial_{i}\left(e_{1}, \ldots, e_{n}\right)^{\tau}=B_{i}\left(e_{1}, \ldots, e_{n}\right)^{\tau} \text { with } B_{i} \in \mathrm{M}_{n}(k), \quad \text { for } i=1, \ldots, m,
$$

induces an integrable system of dimension $n$ of form

$$
\left\{\delta_{1}(Z)=A_{1} Z, \ldots, \delta_{m}(Z)=A_{m} Z\right\}
$$

where $A_{i}=-B_{i}^{\tau}$ for each $i$. The system (3.1) is called the integrable system associated with $M$ w.r.t. a basis $e_{1}, \ldots, e_{n}$. If we choose another basis $f_{1}, \ldots, f_{n}$ of $M$ over $k$ with

$$
\left(f_{1}, \ldots, f_{n}\right)=\left(e_{1}, \ldots, e_{n}\right) T \text { for some } T \in \mathrm{GL}_{n}(k),
$$

then by replacing $Z$ with $T Z^{*}$ in (3.1) we obtain the integrable system for the new basis:

$$
\left\{\delta_{1}\left(Z^{*}\right)=A_{1}^{*} Z^{*}, \quad \ldots, \quad \delta_{m}\left(Z^{*}\right)=A_{m}^{*} Z^{*}\right\},
$$

where $A_{i}^{*}=T^{-1} A_{i} T-T^{-1} \delta_{i}(T)$ for each $i$. Conversely, it is clear that any integrable system of form (3.1) comes from a differential module $M:=k^{n}$ with the canonical basis $\left\{e_{1}, \ldots, e_{n}\right\}$ and the $\partial_{i}$ given by the formulas

$$
\partial_{i}\left(e_{1}, \ldots, e_{n}\right)^{\tau}=-A_{i}^{\tau}\left(e_{1}, \ldots, e_{n}\right)^{\tau} \quad \text { for } i=1, \ldots, m \text {. }
$$

To investigate "solutions" of integrable systems, we introduce

Definition 3.3. A field $K \supseteq k$ is called a differential extension field over $k$ if all derivations $\delta_{1}, \ldots, \delta_{m}$ on $k$ can be extended to $K$ and the extended maps pairwise commute.

Let $K$ be a differential extension field over $k$. A vector $Z^{*} \in K^{n}$ is called a solution of the integrable system (3.1) if $\delta_{i}\left(Z^{*}\right)=A_{i} Z^{*}$ for each $i$.

A nonzero element $h$ of $K$ is said to be hyperexponential over $k$ w.r.t. $\delta_{i}$ if $\frac{\delta_{i}(h)}{h}$ belongs to $k$. The element $h$ is said to be hyperexponential over $k$ if $h$ is hyperexponential over $k$ w.r.t. all $\delta_{i}$. Two hyperexponential elements $h_{1}$ and $h_{2}$ of $K$ are said to be equivalent over $k$ if $\frac{h_{1}}{h_{2}} \in k$; otherwise, $h_{1}$ and $h_{2}$ are said to be inequivalent over $k$. A vector $H \in K^{n}$ is said to be hyperexponential over $k$ 
w.r.t. $\delta_{i}$ if $H$ can be written as $h V$ where $V \in k^{n}$ and $h \in K$ is hyperexponential over $k$ w.r.t. $\delta_{i}$. A vector $H \in K^{n}$ is said to be hyperexponential over $k$ if $H$ is hyperexponential over $k$ w.r.t. all $\delta_{i}$.

Observe that a vector $H \in K^{n}$ is hyperexponential over $k$ if and only if $H$ can be written in form $h V$ where $V \in k^{n}$ and $h \in K$ is hyperexponential over $k$. Indeed, if $H$ is hyperexponential over $k$ then $H=h_{i} V_{i}$ with $V_{i} \in k^{n}$ and $\frac{\delta_{i}\left(h_{i}\right)}{h_{i}} \in k$ for $i=1, \ldots, m$. From $h_{i} V_{i}=h_{j} V_{j}$, it follows that $\frac{h_{i}}{h_{j}} \in k$ for any $i, j$. Hence,

$$
k \ni \delta_{i}\left(\frac{h_{i}}{h_{j}}\right)=\frac{\delta_{i}\left(h_{i}\right) h_{j}-h_{i} \delta_{i}\left(h_{j}\right)}{h_{j}^{2}}=\frac{\delta_{i}\left(h_{i}\right)}{h_{i}} \frac{h_{i}}{h_{j}}-\frac{h_{i}}{h_{j}} \frac{\delta_{i}\left(h_{j}\right)}{h_{j}},
$$

which implies that $\frac{\delta_{i}\left(h_{j}\right)}{h_{j}} \in k$ for each $i$. So, $h_{j}$ is hyperexponential over $k$ and $H=h_{j} V_{j}$ is of desired form.

The following proposition reveals a relation between 1-dimensional submodules of a differential module and hyperexponential solutions of the associated integrable systems. Although this proposition is obvious in the ordinary case, we give a detailed proof here because integrability conditions should be taken into account in the partial case.

Proposition 3.4. Let $M$ be a differential module of dimension $n$ and

$$
\left\{\delta_{1}(Z)=A_{1} Z, \ldots, \delta_{m}(Z)=A_{m} Z\right\}
$$

be the integrable system associated with $M$ w.r.t. a basis $e_{1}, \ldots, e_{n}$. Then, $M$ has a 1-dimensional submodule if and only if (3.2) has a hyperexponential solution.

Proof. Let $H=h V$ be a hyperexponential solution of (3.2) where $h$ is a hyperexponential element of some differential field extension $K$ and $V \in k^{n}$. Set

$$
u=\left(e_{1}, \ldots, e_{n}\right) H \quad \text { and } \quad w=\frac{u}{h}=\left(e_{1}, \ldots, e_{n}\right) V \in M
$$

Since $H$ is a solution of $(3.2)$, we have $\partial_{i}(u)=0$ and

$$
\partial_{i}(w)=\partial_{i}\left(\frac{u}{h}\right)=\frac{\partial_{i}(u) h-\delta_{i}(h) u}{h^{2}}=-\frac{\delta_{i}(h)}{h} \frac{u}{h}=-\frac{\delta_{i}(h)}{h} w \in k w,
$$

for $i=1, \ldots, m$. So, $k w$ is a 1-dimensional submodule of $M$. Suppose that $H$ can also be written as $h_{2} V_{2}$ with $V_{2} \in k^{n}$ and $h_{2} \in K$ hyperexponential over $k$. Set $w_{2}=\left(e_{1}, \ldots, e_{n}\right) V_{2}$. As before, we have

$$
\partial_{i}\left(w_{2}\right)=-\frac{\delta_{i}\left(h_{2}\right)}{h_{2}} w_{2}, \quad i=1, \ldots, m .
$$

From $h V=h_{2} V_{2}$, we have $h=a h_{2}$ for some nonzero $a \in k$ and therefore $V=a^{-1} V_{2}$. So, $k w=k a^{-1} w_{2}=k w_{2}$ and $H$ induces uniquely a 1-dimensional submodule $k w$ of $M$, which is called the 1-dimensional submodule associated with $H$.

Conversely, let $N$ be a 1-dimensional submodule of $M$ generated by $w$ and suppose that $\partial_{i}(w)=a_{i} w$ with $a_{i} \in k$ for $i=1, \ldots, m$. Then,

$$
\partial_{j}\left(\partial_{i}(w)\right)-a_{i} a_{j} w=\delta_{j}\left(a_{i}\right) w
$$


which has a left hand-side invariant under the permutation of $(i, j)$. This implies that $\delta_{j}\left(-a_{i}\right)=\delta_{i}\left(-a_{j}\right)$ for any $i, j$. By [7], there is a well-defined hyperexponential element $h$ over $k$ such that $\delta_{i}(h)=-a_{i} h$ for $i=1, \ldots, m$. Therefore,

$$
\partial_{i}(h w)=0 \text { for } i=1, \ldots, m,
$$

which implies that $h\left(f_{1}, \ldots, f_{n}\right)^{\tau}$ is a solution of the system $(3.2)$ where $f_{1}, \ldots, f_{n}$ are coordinates of $w$ under the basis $e_{1}, \ldots, e_{n}$. Moreover, $h\left(f_{1}, \ldots, f_{n}\right)^{\tau}$ is a hyperexponential vector over $k$ by definition.

Proposition 3.4 reveals that 1-dimensional submodules of a differential module can be constructed via hyperexponential solutions of the associated integrable systems. Note that this construction is independent from the choice of the associated integrable systems. Indeed, let

$$
S: \quad\left\{\delta_{1}(Z)=A_{1} Z, \ldots, \delta_{m}(Z)=A_{m} Z\right\}
$$

and

$$
S^{*}: \quad\left\{\delta_{1}\left(Z^{*}\right)=A_{1}^{*} Z^{*}, \ldots, \delta_{m}\left(Z^{*}\right)=A_{m}^{*} Z^{*}\right\}
$$

be the respective integrable systems associated with $M$ w.r.t. the bases $e_{1}, \ldots, e_{n}$ and $f_{1}, \ldots, f_{n}$. There exists $T \in \mathrm{GL}_{n}(k)$ such that

$$
\left(f_{1}, \ldots, f_{n}\right)=\left(e_{1}, \ldots, e_{n}\right) T .
$$

In addition, $A_{i}^{*}=T^{-1} A_{i} T-T^{-1} \delta_{i}(T)$ for each $i$. One can verify that $T^{-1}$ viewed as a linear transformation from the set of solutions of $S$ to the set of solutions of $S^{*}$ is a bijection. If $H_{1}:=h V$ is a hyperexponential solution of $S$ with $V \in k^{n}$ and $h$ hyperexponential over $k$, then $H_{2}:=T^{-1} H_{1}$ is a hyperexponential solution of $S^{*}$. Therefore, the 1-dimensional submodules of $M$ associated with $H_{1}$ and $H_{2}$ are generated by $\left(e_{1}, \ldots, e_{n}\right) V$ and by $\left(f_{1}, \ldots, f_{n}\right) T^{-1} V$, respectively. From (3.3), these two generators are equal, so are the 1-dimensional submodules they generate.

We now study the structure of all 1-dimensional submodules of differential modules. Let $M$ be a differential module of dimension $n$. Suppose that

$$
S: \quad\left\{\delta_{1}(Z)=A_{1} Z, \ldots, \delta_{m}(Z)=A_{m} Z\right\},
$$

with $A_{i}=\left(a_{i s t}\right) \in \mathrm{M}_{n}(k)$, is the associated integrable system with $M$ w.r.t. a basis $e_{1}, \ldots, e_{n}$, and $M^{*}$ is the dual module of $M$ with the dual basis $\left\{e_{1}^{*}, \ldots, e_{n}^{*}\right\}$ such that

$$
e_{i}^{*}\left(e_{j}\right)=\left\{\begin{array}{cc}
1 & \text { when } i=j, \\
0 & \text { otherwise }
\end{array}\right.
$$

Since $\partial_{i}\left(e_{1}, \ldots, e_{n}\right)^{\tau}=-A_{i}^{\tau}\left(e_{1}, \ldots, e_{n}\right)^{\tau}$ for $i=1, \ldots, m$, we have

$$
\partial_{i}\left(e_{j}^{*}\right)\left(e_{\ell}\right)=-e_{j}^{*}\left(\partial_{i}\left(e_{\ell}\right)\right)+\partial_{i}\left(e_{j}^{*}\left(e_{\ell}\right)\right)=-e_{j}^{*}\left(-\sum_{s=1}^{n} a_{i s \ell} e_{s}\right)=a_{i j \ell},
$$

for $\ell=1, \ldots, n$, hence $\partial_{i}\left(e_{j}^{*}\right)=\sum_{s=1}^{n} a_{i j s} e_{s}^{*}$ for all $i, j$, that is,

$$
\partial_{i}\left(e_{1}^{*}, \ldots, e_{n}^{*}\right)^{\tau}=A_{i}\left(e_{1}^{*}, \ldots, e_{n}^{*}\right)^{\tau}, \quad i=1, \ldots, m .
$$


Thus, $\left(e_{1}^{*}, \ldots, e_{n}^{*}\right)^{\tau}$ is also a solution of the system $S$. Now assume that $k$ contains a nonconstant $a$, i.e., $\partial_{\ell}(a) \neq 0$ for some $\ell$. By Proposition 2.9 in [8], the differential module $M^{*}$ has a cyclic vector $w$ such that $M^{*}$ is generated by $w, \partial_{\ell}(w), \partial_{\ell}^{2}(w), \ldots$ over $k$. So, $M^{*}=k\left[\partial_{\ell}\right] w$ and further $M^{*}=\mathbb{D} w$ because $k\left[\partial_{\ell}\right] \subseteq \mathbb{D}$. Since $\operatorname{dim}_{k} M^{*}=n, w, \partial_{\ell}(w), \ldots, \partial_{\ell}^{n-1}(w)$ form a basis of $M^{*}$ and therefore

$$
\left(e_{1}^{*}, \ldots, e_{n}^{*}\right)^{\tau}=P\left(w, \partial_{\ell}(w), \ldots, \partial_{\ell}^{n-1}(w)\right)^{\tau} \quad \text { for some } P \in \operatorname{GL}_{n}(k) .
$$

In addition, for $i=1, \ldots, m$, the vectors $w, \partial_{i}(w), \partial_{i}^{2}(w), \ldots, \partial_{i}^{n}(w)$ are linearly dependent over $k$ and, therefore, by linear algebra there exists $L_{i} \in k\left[\partial_{i}\right]$ of minimal order such that $L_{i}(w)=0$. This yields the following system of linear PDE's:

$$
L: \quad\left\{L_{1}(y)=0, \quad \ldots, \quad L_{m}(y)=0\right\} .
$$

From (3.4), any hyperexponential solution of $S$ has form

$$
H=P\left(h, \delta_{\ell}(h), \ldots, \delta_{\ell}^{n-1}(h)\right)^{\tau}
$$

for any hyperexponential solution $h$ of $L$. Apply Algorithm HyperexponentialSolutions in [7] to the system $L$, we obtain a finite number of

$$
\begin{array}{cc}
h_{1}, & \left\{r_{11}, \ldots, r_{1, t_{1}}\right\}, \\
\vdots & \vdots \\
h_{s}, & \left\{r_{s 1}, \ldots, r_{s, t_{s}}\right\},
\end{array}
$$

where $h_{1}, \ldots, h_{s}$ are mutually inequivalent hyperexponential elements over $k$ and $r_{i 1}, \ldots, r_{i, t_{i}}$ are elements of $k$ which are linearly independent over $C$. According to Proposition 3.4 in [7], any hyperexponential solution $h$ of $L$ has form

$$
h=h_{i}\left(c_{1} r_{i 1}+\cdots+c_{t_{i}} r_{i, t_{i}}\right) \text { for some } i \in\{1, \ldots, s\} \text {, }
$$

with the $c_{j}$ arbitrary constants of $k$, not all zero. For $i=1, \ldots, s$ and $j=1, \ldots, t_{i}$,

$$
P\left(h_{i} r_{i j}, \delta_{\ell}\left(h_{i} r_{i j}\right), \ldots, \delta_{\ell}^{n-1}\left(h_{i} r_{i j}\right)\right)^{\tau}=h_{i} V_{i j} \quad \text { for some } V_{i j} \in k^{n} .
$$

Therefore, any hyperexponential solution $H$ of $S$ has form

$$
H=h_{i}\left(c_{1} V_{i 1}+\cdots+c_{t_{i}} V_{i, t_{i}}\right) \quad \text { for some } i \in\{1, \ldots, s\},
$$

where the $c_{j}$ are arbitrary constants of $k$, not all zero. Since $r_{i 1}, \ldots, r_{i, t_{i}}$ are linearly independent over $C$, so are the vectors $V_{i 1}, \ldots, V_{i, t_{i}}$. Set

$$
N_{i}=k\left(e_{1}, \ldots, e_{n}\right)\left(c_{1} V_{i 1}+\cdots+c_{t_{i}} V_{i, t_{i}}\right), \quad i=1, \ldots, s,
$$

for arbitrarily chosen constants $c_{1}, \ldots, c_{t_{i}}$ of $k$, not all zero. From the proof of Proposition 3.4, $N_{1}, \ldots, N_{s}$ are 1-dimensional submodules of $M$. Furthermore, we will show in the sequel that

$I_{i}=\left\{N_{i} \mid c_{1}, \ldots, c_{t_{i}}\right.$ arbitrary constants of $k$, not all zero $\}, \quad i=1, \ldots, s$, constitute a partition of the set of all 1-dimensional submodules of $M$ by the equivalence relation " $\simeq$ ", the isomorphism between differential modules. 
Indeed, we first show that each $I_{i}$ is a well-defined equivalence class w.r.t. " $\simeq$ ". Suppose that $N_{i}$ and $N_{i}^{\prime}$ are two 1-dimensional submodules of $M$ belonging to $I_{i}$. Then, there are two set of constants $c_{1}, \ldots, c_{t_{i}}$ and $c_{1}^{\prime}, \ldots, c_{t_{i}}^{\prime}$ of $k$, not all zero, such that

$$
N_{i}=k \underbrace{\left(e_{1}, \ldots, e_{n}\right)\left(c_{1} V_{i 1}+\cdots+c_{t_{i}} V_{i, t_{i}}\right)}_{w_{i}},
$$

and

$$
N_{i}^{\prime}=k \underbrace{\left(e_{1}, \ldots, e_{n}\right)\left(c_{1}^{\prime} V_{i 1}+\cdots+c_{t_{i}}^{\prime} V_{i, t_{i}}\right)}_{w_{i}^{\prime}} .
$$

From the previous discussion, we have

$$
\partial_{j}\left(w_{i}\right)=-\frac{\delta_{j}\left(h_{i}\right)}{h_{i}} w_{i} \quad \text { and } \quad \partial_{j}\left(w_{i}^{\prime}\right)=-\frac{\delta_{j}\left(h_{i}\right)}{h_{i}} w_{i}^{\prime}, \quad j=1, \ldots, m .
$$

Clearly, $N_{i}=k w_{i} \simeq N_{i}^{\prime}=k w_{i}^{\prime}$ where the isomorphism is given by $w_{i} \mapsto w_{i}^{\prime}$. So, $I_{i}$ is a well-defined equivalence class. Let $k w$ be a 1-dimensional submodule of $M$. Suppose that $w=\left(e_{1}, \ldots, e_{n}\right) V$ with $V \in k^{n}$. From the proof of Proposition 3.4, there exists a hyperexponential element $h$ such that $h V$ is a hyperexponential solution of $S$. Therefore,

$$
h V=h_{i}\left(c_{1} V_{i 1}+\cdots+c_{t_{i}} V_{i, t_{i}}\right),
$$

for some $i \in\{1, \ldots, s\}$ and some constants $c_{1}, \ldots, c_{t_{i}}$ of $k$, not all zero. From (3.5), we know that $h$ and $h_{i}$ are equivalent over $k$ and $h=a h_{i}$ for some $a \in k$. Thus, $V=a^{-1}\left(c_{1} V_{i 1}+\cdots+c_{t_{i}} V_{i, t_{i}}\right)$ and

$$
k w=k\left(e_{1}, \ldots, e_{n}\right) V=k\left(e_{1}, \ldots, e_{n}\right)\left(c_{1} V_{i 1}+\cdots+c_{t_{i}} V_{i, t_{i}}\right)
$$

for some constants $c_{1}, \ldots, c_{t_{i}}$ of $k$. So, $k w$ belongs to the equivalence class $I_{i}$. Let $N_{i}$ and $N_{j}$ be two 1-dimensional submodules in the equivalence classes $I_{i}$ and $I_{j}$, respectively. Then, there are two sets of constants $c_{1}, \ldots, c_{t_{i}}$ and $c_{1}^{\prime}, \ldots, c_{t_{j}}^{\prime}$ of $k$, both not all zero, such that

$$
N_{i}=k \underbrace{\left(e_{1}, \ldots, e_{n}\right)\left(c_{1} V_{i 1}+\cdots+c_{t_{i}} V_{i, t_{i}}\right)}_{w_{i}},
$$

and

$$
N_{j}=k \underbrace{\left(e_{1}, \ldots, e_{n}\right)\left(c_{1}^{\prime} V_{j 1}+\cdots+c_{t_{j}}^{\prime} V_{j, t_{j}}\right)}_{w_{j}} .
$$

Suppose that $N_{i} \simeq N_{j}$ given by the map $\phi: w_{i} \mapsto a w_{j}$ with $a \in k$. Then, $\partial_{l} \circ \phi\left(w_{i}\right)=\phi \circ \partial_{l}\left(w_{i}\right)$ together with the relation that $\partial_{l}\left(w_{i}\right)=-\frac{\delta_{l}\left(h_{i}\right)}{h_{i}} w_{i}$ and $\partial_{l}\left(w_{j}\right)=-\frac{\delta_{l}\left(h_{j}\right)}{h_{j}} w_{j}$ for $l=1, \ldots, m$, implies that $\frac{h_{i}}{h_{j}} \in k$, a contradiction with the fact that $h_{i}$ and $h_{j}$ are inequivalent over $k$. So, $\left\{I_{1}, \ldots, I_{s}\right\}$ is a partition of all 1-dimensional submodules of $M$ given by the equivalence relation " $\simeq$ ".

We now describe an algorithm for finding 1-dimensional submodules of differential modules. 
Algorithm OneDimSubMods (Find 1-dimensional submodules of a differential module)

Input: A differential module $M$ with a basis $e_{1}, \ldots, e_{n}$ and the action of $\partial_{i}$ on this basis:

$$
\partial_{i}\left(e_{1}, \ldots, e_{n}\right)^{\tau}=B_{i}\left(e_{1}, \ldots, e_{n}\right)^{\tau}, \quad i=1, \ldots, m,
$$

where $B_{1}, \ldots, B_{m}$ are $n \times n$ matrices with entries in $k$.

Output: All 1-dimensional submodules of $M$.

1. [find the cyclic vector] Construct the integrable system associated with $M$

$$
S: \quad\left\{\delta_{1}(Z)=A_{1} Z, \quad \ldots, \delta_{m}(Z)=A_{m} Z\right\}
$$

where $A_{i}=-B_{i}^{\tau}$. Let $M^{*}$ be the dual module of $M$ with the dual basis $\left\{e_{1}^{*}, \ldots, e_{n}^{*}\right\}$ over $k$ such that

$$
\partial_{i}\left(e_{1}^{*}, \ldots, e_{n}^{*}\right)^{\tau}=A_{i}\left(e_{1}^{*}, \ldots, e_{n}^{*}\right)^{\tau}, \quad i=1, \ldots, m .
$$

Find a cyclic vector $w$ of $M^{*}$ such that $M^{*}$ is generated by $w, \partial_{\ell}(w), \ldots, \partial_{\ell}^{n-1}(w)$ over $k$ for some $\ell \in\{1, \ldots, m\}$. Then, there exists $P \in \mathrm{GL}_{n}(k)$ such that

$$
\left(e_{1}^{*}, \ldots, e_{n}^{*}\right)^{\tau}=P\left(w, \partial_{\ell}(w), \ldots, \partial_{\ell}^{n-1}(w)\right)^{\tau} .
$$

By linear algebra, find linear ordinary operators $L_{i} \in k\left[\partial_{i}\right]$ of minimal order such that $L_{i}(w)=0$ for $i=1, \ldots, m$. We then obtain a system of linear PDE's:

$$
L: \quad\left\{L_{1}(y)=0, \quad \ldots, \quad L_{m}(y)=0\right\} .
$$

2. [compute hyperexponential solutions] Apply Algorithm HyperexponentialSolutions in [7] to compute all hyperexponential solutions of $L$. If the output returns NULL then exit [ $M$ has no 1-dimensional submodules]. Otherwise, we obtain

$$
\begin{array}{cc}
h_{1}, & \left\{r_{11}, \ldots, r_{1, t_{1}}\right\}, \\
\vdots & \vdots \\
h_{s}, & \left\{r_{s 1}, \ldots, r_{s, t_{s}}\right\},
\end{array}
$$

where $h_{1}, \ldots, h_{s}$ are mutually inequivalent hyperexponential elements over $k$ and $r_{i 1}, \ldots, r_{i, t_{i}} \in k$ are linearly independent over $C$. Set

$$
V_{i j}=h_{i}^{-1} P\left(h_{i} r_{i j}, \delta_{\ell}\left(h_{i} r_{i j}\right), \ldots, \delta_{\ell}^{n-1}\left(h_{i} r_{i j}\right)\right)^{\tau} \in k^{n},
$$

for $i=1, \ldots, s$ and $j=1, \ldots, t_{i}$. Then, $V_{i 1}, \ldots, V_{i, t_{i}}$ are linearly independent over $C$ and

$$
\begin{array}{cc}
h_{1}, & \left\{V_{11}, \ldots, V_{1, t_{1}}\right\}, \\
\vdots & \vdots \\
h_{s}, & \left\{V_{s 1}, \ldots, V_{s, t_{s}}\right\},
\end{array}
$$

describes the structure of hyperexponential solutions of $S$ : any hyperexponential solution of $S$ has form:

$$
H=h_{i}\left(c_{1} V_{i 1}+\cdots+c_{t_{i}} V_{i, t_{i}}\right),
$$

with $i \in\{1, \ldots, s\}$ and the $c_{j}$ constants of $k$, not all zero. 
3. [retrieve 1-dimensional submodules] Set

$$
\begin{aligned}
I_{i}= & \left\{k\left(e_{1}, \ldots, e_{n}\right)\left(c_{1} V_{i 1}+\cdots+c_{t_{i}} V_{i, t_{i}}\right) \mid c_{1}, \ldots, c_{t_{i}}\right. \text { arbitrary constants } \\
& \text { of } k, \text { not all zero }\}, \quad i=1, \ldots, m .
\end{aligned}
$$

Then, $\left\{I_{1}, \ldots, I_{s}\right\}$ is a partition of all 1-dimensional submodules of $M$ by the equivalence relation " $\simeq$ ".

\subsection{Decomposability of Elements of $\wedge^{d} M$}

Let $M$ be a differential module. In order to find all $d$-dimensional submodules of $M$, by Proposition 3.1 it suffices to find all those 1-dimensional submodules of $\wedge^{d} M$ whose generators are decomposable.

Applying the algorithm OneDimSubMods to $\wedge^{d} M$ yields all its 1-dimensional submodules $k w$ where $w \in \wedge^{d} M$ may contain some unspecified constants $c_{1}, \ldots, c_{t}$. To test the decomposability of $w$, consider the map

$$
\phi_{w}: M \rightarrow \wedge^{d+1} M, \quad v \mapsto v \wedge w .
$$

From the proof of Proposition 3.1, $w$ is decomposable if and only if $\operatorname{ker}\left(\phi_{w}\right)$ is of dimension $d$, while the latter is equivalent to the condition that the matrix $P$ of the $\phi_{w}$ has rank $n-d$. Hence, identifying the decomposability of $w$ amounts to a rank computation, i.e., identifying the constants $c_{1}, \ldots, c_{t}$ in $w$ such that all $(n-d+1) \times(n-d+1)$ minors of $P$ are zero and there exists at least a nonzero $(n-d) \times(n-d)$ minor. We observe that this is exactly the Plücker relations described in [12]. Solving the Plücker relations amounts to solving a nonlinear system in $c_{1}, \ldots, c_{t}$. If such a system has no solutions in $\bar{C}$, the algebraic closure of $C$, then $M$ has no $d$-dimensional submodules. Otherwise, $\operatorname{ker}\left(\phi_{w}\right)$ is a $d$-dimensional submodule of $M$.

Remark 3.5. There are alternative ways to compute ranks of parameterized matrices, for example, the Gauss method with branching, a Gröbner basis method using the linear structure [4] or the algorithm described in [11] for computing the rank of parameterized linear systems. These methods may be more efficient than computing minors.

\section{Factorization Algorithm}

Based on the results in previous sections, we now describe an algorithm for factoring differential modules.

Algorithm FactorDiffMod (Factor differential modules)

Input: A differential module $M$ with a basis $\left\{e_{1}, \ldots, e_{n}\right\}$ and the action of $\partial_{i}$ on this basis:

$$
\partial_{i}\left(e_{1}, \ldots, e_{n}\right)^{\tau}=B_{i}\left(e_{1}, \ldots, e_{n}\right)^{\tau}, \quad \text { for } i=1, \ldots, m,
$$

where $B_{1}, \ldots, B_{m}$ are $n \times n$ matrices with entries in $k$.

Output: For $d<n$, all $d$-dimensional submodules of $M$ given by their bases and the action of $\partial_{i}$ on the bases. 
1. [construct the exterior power] From (4.1), construct a basis $\left\{f_{1}, \ldots, f_{\tilde{N}}\right\}$ of $\wedge^{d} M$ with $\tilde{N}=\left(\begin{array}{c}n \\ d\end{array}\right)$ and the matrices $\tilde{B}_{i} \in \mathrm{M}_{\tilde{N}}(k)$ such that

$$
\partial_{i}\left(f_{1}, \ldots, f_{\tilde{N}}\right)^{\tau}=\tilde{B}_{i}\left(f_{1}, \ldots, f_{\tilde{N}}\right)^{\tau}, \quad i=1, \ldots, m .
$$

2. [compute 1-dimensional submodules] Apply the algorithm OneDimSubMods to compute all 1-dimensional submodules of $\wedge^{d} M$. If $\wedge^{d} M$ has no 1-dimensional submodules, then exit [ $M$ has no $d$-dimensional submodules]. Otherwise, suppose that $k w$ is a 1-dimensional submodule of $\wedge^{d} M$ where $w$ may contain some unspecified constants $c_{1}, \ldots, c_{t}$ of $k$.

3. [test the decomposability] For each $w$ obtained in Step 2, consider the map

$$
\phi_{w}: M \rightarrow \wedge^{d+1} M, \quad v \mapsto v \wedge w
$$

Construct the matrix $P$ of $\phi_{w}$, which is an $\left(\begin{array}{c}n \\ d+1\end{array}\right) \times n$ matrix with entries in $k\left(c_{1}, \ldots, c_{t}\right)$. Compute all $(n-d+1) \times(n-d+1)$ minors of $P$. Equating these minors to zero yields a nonlinear system in $c_{1}, \ldots, c_{t}$. If this nonlinear system has no solutions in $\bar{C}$ then exit [ $M$ has no $d$-dimensional submodules]. Otherwise, substitute the values of $c_{1}, \ldots, c_{t}$ into $P$ and compute a basis $\left\{\alpha_{1}, \ldots, \alpha_{d}\right\}$ of the rational kernel of $P$ with $\alpha_{j} \in k^{n}$.

4. [retrieve $d$-dimensional submodules] Set $v_{j}=\left(e_{1}, \ldots, e_{n}\right) \alpha_{j}$ for $j=1, \ldots, d$. Then, $\oplus_{j=1}^{d} k v_{j}$ is a $d$-dimensional submodule of $M$.

We now apply the algorithm FactorDiffMod to redo Example 1 in [6].

Example. Let $\mathbb{D}=\overline{\mathbb{Q}}(x, y)\left[\partial_{x}, \partial_{y}\right]$ where $\partial_{x}=\frac{\partial}{\partial x}$ and $\partial_{y}=\frac{\partial}{\partial y}$ are the usual differential operators w.r.t. $x$ and $y$, respectively. Let $M$ be a differential module with a basis $\left\{e_{1}, e_{2}, e_{3}\right\}$ such that

$$
\partial_{x}\left(e_{1}, e_{2}, e_{3}\right)^{\tau}=B_{x}\left(e_{1}, e_{2}, e_{3}\right)^{\tau}, \quad \partial_{y}\left(e_{1}, e_{2}, e_{3}\right)^{\tau}=B_{y}\left(e_{1}, e_{2}, e_{3}\right)^{\tau}
$$

where

$$
B_{x}=\left(\begin{array}{ccc}
0 & -\frac{y}{4 x} & -\frac{1}{4} \\
-1 & \frac{2-x y}{4 x} & -\frac{2+x y}{4 y} \\
0 & \frac{y^{2}}{4 x} & \frac{y}{4}
\end{array}\right), \quad B_{y}=\left(\begin{array}{ccc}
0 & -\frac{1}{4} & -\frac{x}{4 y} \\
0 & -\frac{2+x y}{4 y} & \frac{2 x-x^{2} y}{4 y^{2}} \\
-1 & \frac{y}{4} & \frac{x}{4}
\end{array}\right) .
$$

To compute two-dimensional submodules of $M$, construct the second exterior power $\wedge^{2} M$ of $M$ with a basis $\left\{f_{1}:=e_{1} \wedge e_{2}, f_{2}:=e_{1} \wedge e_{3}, f_{3}:=e_{2} \wedge e_{3}\right\}$ and compute the integrable system associated with $\wedge^{2} M$ :

$$
S: \quad\left\{\partial_{x}(Z)=A_{x x} Z, \quad \partial_{y}(Z)=A_{y y} Z\right\}
$$


where $Z=\left(z_{1}, z_{2}, z_{3}\right)^{\tau}$ is a vector of unknowns and

$$
A_{x x}=\left(\begin{array}{ccc}
\frac{x y-2}{4 x} & -\frac{y^{2}}{4 x} & 0 \\
\frac{2+x y}{4 y} & -\frac{y}{4} & 1 \\
-\frac{1}{4} & \frac{y}{4 x} & -\frac{1}{2 x}
\end{array}\right), \quad A_{y y}=\left(\begin{array}{ccc}
\frac{2+x y}{4 y} & -\frac{y}{4} & -1 \\
\frac{x^{2} y-2 x}{4 y^{2}} & -\frac{x}{4} & 0 \\
-\frac{x}{4 y} & \frac{1}{4} & \frac{1}{2 y}
\end{array}\right) .
$$

Let $M_{2}^{*}$ be the dual module of $\wedge^{2} M$ with the dual basis $\left\{f_{1}^{*}, f_{2}^{*}, f_{3}^{*}\right\}$. Then,

$$
\partial_{x}\left(f_{1}^{*}, f_{2}^{*}, f_{3}^{*}\right)^{\tau}=A_{x x}\left(f_{1}^{*}, f_{2}^{*}, f_{3}^{*}\right)^{\tau} \quad \text { and } \quad \partial_{y}\left(f_{1}^{*}, f_{2}^{*}, f_{3}^{*}\right)^{\tau}=A_{y y}\left(f_{1}^{*}, f_{2}^{*}, f_{3}^{*}\right)^{\tau} \text {. }
$$

We find that $f_{1}^{*}$ is a cyclic vector of $M_{2}^{*}$ and moreover,

$$
\left(f_{1}^{*}, f_{2}^{*}, f_{3}^{*}\right)^{\tau}=P\left(f_{1}^{*}, \partial_{x}\left(f_{1}^{*}\right), \partial_{x}^{2}\left(f_{1}^{*}\right)\right)^{\tau}
$$

with

$$
P=\left(\begin{array}{ccc}
1 & 0 & 0 \\
\frac{x y-2}{4 x} & -\frac{y^{2}}{4 x} & 0 \\
-\frac{3(x y-2)}{8 x^{2}} & \frac{3 y^{2}}{8 x^{2}} & -\frac{y^{2}}{4 x}
\end{array}\right) .
$$

By linear algebra, we find two linear ordinary differential operators, both of minimal order,

and

$$
L_{x}=\partial_{x}^{3}+\frac{3}{x} \partial_{x}^{2}+\frac{3-x y}{4 x^{2}} \partial_{x}-\frac{y}{8 x^{2}} \in \overline{\mathbb{Q}}(x, y)\left[\partial_{x}\right]
$$

$$
L_{y}=\partial_{y}^{3}+\frac{6-2 x y}{y(x y-6)} \partial_{y}^{2}+\frac{23 x y-x^{2} y^{2}-42}{4 y^{2}(x y-6)} \partial_{y}+\frac{x^{2} y^{2}-30 x y+72}{8 y^{3}(x y-6)} \in \overline{\mathbb{Q}}(x, y)\left[\partial_{y}\right]
$$

such that $L_{x}$ and $L_{y}$ annihilate $f_{1}^{*}$.

Apply Algorithm HyperexponentialSolutions in [7] to the system

$$
L: \quad\left\{L_{x}\left(z_{1}\right)=0, \quad L_{y}\left(z_{1}\right)=0\right\},
$$

we find that any hyperexponential solution $h$ of the system $L$ has form

$$
h=c e^{\int\left(-\frac{1}{2 x} d x+\frac{1}{2 y} d y\right)}=c \sqrt{\frac{y}{x}}, \quad \text { for any } c \in \overline{\mathbb{Q}},
$$

where $e^{\int\left(-\frac{1}{2 x} d x+\frac{1}{2 y} d y\right)}$ denotes a hyperexponential function $h_{0}$ in $x, y$ such that

$$
\partial_{x}\left(h_{0}\right)=-\frac{1}{2 x} h_{0} \quad \text { and } \quad \partial_{y}\left(h_{0}\right)=\frac{1}{2 y} h_{0} .
$$

Hence, any hyperexponential solution of $S$ has form

$$
Z=P\left(h, \partial_{x}(h), \partial_{x}^{2}(h)\right)^{\tau}=\sqrt{\frac{y}{x}}\left(c, c \cdot \frac{x}{y}, 0\right)^{\tau}, \quad \text { for any } c \in \overline{\mathbb{Q}},
$$

and all 1-dimensional submodules of $\wedge^{2} M$ are of form $\overline{\mathbb{Q}}(x, y) w$ where

$$
w=\left(f_{1}, f_{2}, f_{3}\right)\left(1, \frac{x}{y}, 0\right)^{\tau}=e_{1} \wedge e_{2}+\frac{x}{y} e_{1} \wedge e_{3} \in \wedge^{2} M
$$


To test the decomposability of $w$, consider the map

$$
\phi_{w}: M \rightarrow \wedge^{3} M, \quad v \mapsto v \wedge w
$$

The matrix of $\phi_{w}$ is $P=\left(0,-\frac{x}{y}, 1\right)$ and then has rank one. So, $w$ is decomposable. (In fact, from (4.2) one can see directly that $w=e_{1} \wedge\left(e_{2}+\frac{x}{y} e_{3}\right)$ is a decomposable element of $\wedge^{2}$ M.) A basis for the rational kernel of $P$ is:

$$
\left\{(1,0,0)^{\tau}, \quad\left(0,1, \frac{x}{y}\right)^{\tau}\right\} \text {. }
$$

Set $v_{1}=\left(e_{1}, e_{2}, e_{3}\right)(1,0,0)^{\tau}=e_{1}$ and $v_{2}=\left(e_{1}, e_{2}, e_{3}\right)\left(0,1, \frac{x}{y}\right)^{\tau}=e_{2}+\frac{x}{y} e_{3}$. Then, $\operatorname{ker}\left(\phi_{u}\right)=k v_{1} \oplus k v_{2}$ is a two-dimensional submodule of $M$ and the actions of $\partial_{x}$ and $\partial_{y}$ on the basis $\left\{v_{1}, v_{2}\right\}$ are given by

$$
\partial_{x}\left(v_{1}, v_{2}\right)^{\tau}=F_{x}\left(v_{1}, v_{2}\right)^{\tau}, \quad \partial_{y}\left(v_{1}, v_{2}\right)^{\tau}=F_{y}\left(v_{1}, v_{2}\right)^{\tau}
$$

where

$$
F_{x}=\left(\begin{array}{cc}
0 & -\frac{y}{4 x} \\
-1 & \frac{1}{2 x}
\end{array}\right), \quad F_{y}=\left(\begin{array}{cc}
0 & -\frac{1}{4} \\
-\frac{x}{y} & -\frac{1}{2 y}
\end{array}\right)
$$

\section{Conclusion and Future Work}

In this paper, we present an algorithm for factoring differential modules. By factoring the differential modules associated with systems of linear PDE's with finitedimensional solution spaces, the algorithm FactorDiffMod improves the factorization algorithm in [6]. Further work will include the refinement of the step in the algorithm OneDimSubMods which deals with computing hyperexponential solutions of integrable systems and the improvement for computing ranks of parameterized matrices. The generalization of the factorization algorithm to difference modules and differential-difference modules will also be studied.

\section{References}

[1] E. Beke. Die Irreduzibilität der homogenen linearen Differentialgleichungen. Mathematische Annalen, 45:278-294, 1894.

[2] M. Bronstein. An improved algorithm for factoring linear ordinary differential operators. In Proc. Int. Symp. on Symbolic Algebraic Comput. (ISSAC'1992), pages 336-340. M. Giesbrecht (ed.), ACM Press, 1992.

[3] E. Compoint and J. A. Weil. Absolute reducibility of differential operators and Galois groups. J. of Algebra, Vol 275/1, pp 77-105, 2004.

[4] D. Cox, J. Little and D. O'Shea. Ideals, Varieties, and Algorithms. Springer, 1996.

[5] Z. Li, F. Schwarz. Rational solutions of Riccati-like partial differential equations. $J$. Symb. Comput., 31:691-716, 2001. 
[6] Z. Li, F. Schwarz and S. Tsarev. Factoring zero-dimensional ideals of linear partial differential operators. In Proc. Int. Symp. on Symbolic Algebraic Comput. (ISSAC'2002), pages 168-175. T. Mora (ed.), ACM Press, 2002.

[7] Z. Li, F. Schwarz and S. Tsarev. Factoring systems of linear PDE's with finitedimensional solution spaces. J. Symb. Comput., 36:443-471, 2003.

[8] M. van der Put and M. F. Singer. Galois Theory of Linear Differential Equations, vol. 328 of Grundlehren der Mathematischen Wissenschaften. Springer, Heidelberg, 2003.

[9] L. Schlesinger. Handbuch der Theorie der Linearen Differentialgleichungen. Teubner, Leipzig, 1895.

[10] F. Schwarz. A factorization algorithm for linear ordinary differential equations. In Proc. Int. Symp. on Symbolic Algebraic Comput. (ISSAC'89), pages 17-25. G. Gonnet (ed.), ACM Press, 1989.

[11] W. Y. Sit. A theory for parametric linear systems. In Proc. Int. Symp. on Symbolic Algebraic Comput. (ISSAC'91), pages 112-121. S. M. Watt (ed.), ACM Press, 1991.

[12] S. P. Tsarev. Problems that appear during factorization of ordinary linear differential operators. Program. Comput. Softw., 20(1):27-29, 1994.

\section{Acknowledgment}

I am indebted to my two co-advisors Prof. Manuel Bronstein and Prof. Ziming Li for their invaluable instructions and advices on my writing and revising the paper. I would like also to thank the referee for helpful suggestions on the algorithmic consideration of our work.

Min $\mathrm{Wu}$

Key Lab of Mathematics-Mechanization Academy of Mathematics and System Sciences

Zhong Guan Cun, Beijing (100080), China

e-mail: wumin@amss.ac.cn 